\title{
Atmospheric neutrino anomaly without maximal mixing?
}

\author{
A. De Rújula* \\ Theory Division, CERN, 1211 Geneva 23, Switzerland \\ M. B. Gavela ${ }^{\dagger}$ \\ Departamento de Física Teórica, Universidad Autónoma de Madrid, Spain \\ P. Hernández \\ Theory Division, CERN, 1211 Geneva 23, Switzerland
}

(Received 7 June 2000; published 5 January 2001)

\begin{abstract}
We consider a pattern of neutrino masses in which there is an approximate mass degeneracy between the two mass eigenstates most coupled to the $\nu_{\mu}$ and $\nu_{\tau}$ flavor eigenstates. Earth-matter effects can lift this degeneracy and induce an effectively maximal mixing between these two generations. This occurs if $\nu_{e}$ 's contain comparable admixtures of the degenerate eigenstates, even if they are not large. This provides an explanation of the atmospheric neutrino anomaly in which the $a b$ initio introduction of a large mixing angle is not required. To test this possibility we perform a detailed analysis of the 52 kiloton-year SuperKamiokande data, and we find that in a large region of parameter space the corresponding confidence levels are excellent. The most recent results from the $\mathrm{CHOOZ}$ reactor experiment, however, severely curtail this region, so that the conventional scenario with nearly maximal mixing angles, which we also analyze in detail, is supported by the data.
\end{abstract}

DOI: 10.1103/PhysRevD.63.033001

PACS number(s): 14.60.Pq

\section{INTRODUCTION}

The results of the SuperKamiokande (SK) Collaboration on the atmospheric neutrino deficit [1] can be explained in terms of neutrino oscillations [2]. It is natural to analyze the data in the context of the most general mixing pattern of three neutrinos, since that is their known number. Three generations are necessary if oscillations are to explain the atmospheric and solar [3] anomalies: a scheme with only two neutrinos cannot account for both effects.

Let $\bar{U}$, with $\left(\nu_{e}, \nu_{\mu}, \nu_{\tau}\right)^{T}=\bar{U} \cdot\left(\nu_{1}, \nu_{2}, \nu_{3}\right)^{T}$, be the Cabibbo-Kobayashi-Maskawa (CKM) matrix in its most conventional parametrization, reviewed by the Particle Data Group [4]:

$$
\begin{aligned}
\bar{U} \equiv & \bar{U}_{23} \bar{U}_{13} \bar{U}_{12} \equiv\left(\begin{array}{ccc}
1 & 0 & 0 \\
0 & \bar{c}_{23} & \bar{s}_{23} \\
0 & -\bar{s}_{23} & \bar{c}_{23}
\end{array}\right) \\
& \times\left(\begin{array}{ccc}
\bar{c}_{13} & 0 & \bar{s}_{13} e^{i \delta} \\
0 & 1 & 0 \\
-\bar{s}_{13} e^{-i \delta} & 0 & \bar{c}_{13}
\end{array}\right)\left(\begin{array}{ccc}
\bar{c}_{12} & \bar{s}_{12} & 0 \\
-\bar{s}_{12} & \bar{c}_{12} & 0 \\
0 & 0 & 1
\end{array}\right)
\end{aligned}
$$

with $\bar{s}_{12} \equiv \sin \bar{\theta}_{12}$, and similarly for the other sines and cosines. Several groups have performed analyses of atmospheric and solar data in terms of three-neutrino mixing [5],

\footnotetext{
*Email address: derujula@nxth21.cern.ch

${ }^{\dagger}$ Email address: gavela@garuda.ft.uam.es

*Email address: pilar.hernandez@cern.ch. On leave from Dept. de Física Teórica, Universidad de Valencia.
}

as described by Eq. (1), or including sterile neutrinos [6]. It is common to these studies to restrict to a "minimal scheme," in which the mass-square difference relevant to atmospheric oscillations dominates over the one relevant to solar neutrinos: $\Delta \bar{m}_{23}^{2} \gg \Delta \bar{m}_{12}^{2}$. In this scenario, the number of parameters describing oscillations at terrestrial distances is reduced to three, $\bar{s}_{13}, \bar{s}_{23}$, and $\Delta \bar{m}_{23}^{2}$, while those most relevant to solar neutrinos are $\bar{s}_{12}, \bar{s}_{13}$, and $\Delta \bar{m}_{12}^{2}$. The best fit of the atmospheric data [7] is

$$
\Delta \bar{m}_{23}^{2} \sim 2.8 \times 10^{-3} \mathrm{eV}^{2}, \quad \sin ^{2}\left(2 \bar{\theta}_{23}\right) \sim 1, \quad \bar{s}_{13}^{2} \sim 2 \times 10^{-2} .
$$

The angle $\bar{\theta}_{23} \simeq \pi / 4$ is close to maximal, to explain the dearth of muons in the SK data.

The situation for solar neutrino oscillations is less definite [8]. The combined solar experiments allow for three different regions of parameter space. The solar deficit can be interpreted either as Mikheyev-Smirnov-Wolfenstein (MSW) (matter enhanced) oscillations [9] with an angle $\bar{\theta}_{12}$ that can be large or small, or as nearly maximal vacuum oscillations, $\bar{\theta}_{12} \simeq \pi / 4$. The corresponding mass differences, $\Delta \bar{m}_{12}^{2}$ $=10^{-6}$ to $10^{-4} \mathrm{eV}^{2}$, or some $10^{-10} \mathrm{eV}^{2}$, are significantly below the range deduced from atmospheric observations, giving support to the minimal scheme. If $\Delta \bar{m}_{12}^{2}$ $\sim O\left(10^{-3}\right) \mathrm{eV}^{2}$, it can have non-negligible effects on atmospheric data that have been recently studied [10].

As is well known, the field of neutrino oscillations is permeated by a tally of implausible facts and coincidences. Oscillations over a distance $L$ occur if $\Delta m^{2} L / E_{\nu} \sim 1$. In the various data samples used by the SK Collaboration, the average neutrino energies are roughly 1,10 , and $100 \mathrm{GeV}$, so that for the value of $\Delta m_{23}^{2}$ in Eq. (2), the "right" distances to 
measure an effect are 280, 2800, and $28000 \mathrm{~km}$ : the size of our planet and the energies in the cosmic ray spectrum have been chosen snugly. Something entirely similar can be said about the low-mass or "just-so" solution to the solar neutrino problem. Moreover, the solar and atmospheric neutrino "anomalies" could have been observed only if the effects are large. This requires surprisingly large mixing angles, except for the "small-angle" MSW solution to the solar neutrino problem, for which the ambient matter elegantly enhances the effect of a small vacuum mixing. Considerable theoretical effort has been invested in arguing that large neutrino mixings are natural, as small quark mixings are believed to be.

A final peculiarity of the observed atmospheric neutrino oscillations involves the matter contribution to the effective squared mass of electron neutrinos:

$$
A \equiv 2 \sqrt{2} G_{F} E_{\nu} n_{e},
$$

where $n_{e}$ is the electron number density in the Earth. For a typical average terrestrial density of $5 \mathrm{~g} / \mathrm{cm}^{3}$ and $E_{\nu}$ $=10 \mathrm{GeV}, \quad A \sim 3.7 \times 10^{-3} \mathrm{eV}^{2}$, again in the ballpark of Eq. (2). This last coincidence suggests the existence of a "small-angle solution" to the atmospheric neutrino problem.

In the "small-angle" scheme we study, the large observed $\nu_{\mu}$ disappearance is generated in the following way. Let $\nu_{2}$ and $\nu_{3}$ be almost degenerate, and heavier than $\nu_{1}$. When neutrinos traverse the Earth, the degeneracy is lifted by matter effects, enhancing $\nu_{\mu}-\nu_{\tau}$ oscillations [11]. We find that the consequent transitions are maximal if the electron neutrino has comparable vacuum admixtures of the degenerate mass eigenstates, $\bar{U}(e 2) \simeq \bar{U}(e 3)$, even if these quantities are not large.

We perform an analysis of atmospheric zenith angle data in the context of three-family mixing with one scale dominance as that in [7], and find that an explanation of the atmospheric neutrino anomaly not involving nearly-maximal neutrino mixing indeed exists, but is disfavored by the complementary information from reactor neutrinos. For the current experimental situation, nearly maximal neutrino mixing angles seem to be unavoidable. The titles of the chapters and appendixes specify the structure of this paper.

\section{APPARENT LARGE MIXINGS INDUCED BY MATTER EFFECTS}

\section{A. From small to large angles}

In a three-family scenario, let one neutrino mass eigenstate be much lighter than the other nearly-degenerate two. Their squared mass matrix can be written as

$$
M_{K}^{v a c} \simeq \operatorname{Diag}\left[\mu_{1}^{2} ; m^{2}+\mu_{2}^{2} ; m^{2}+\mu_{3}^{2}\right],
$$

where $K$ stands for the "kinetic" eigenbasis (as opposed to the flavor basis), vac is for vacuum, and $\mu_{i}^{2} \ll m^{2}$. The degree of degeneracy assumed for the two heavier neutrinos embodies three conditions: their mass difference $\Delta m_{23}^{2} \equiv \mu_{3}^{2}$ $-\mu_{2}^{2}$ is much smaller than their common mass scale $m^{2}$; it is also small enough not to induce observable oscillations over terrestrial distances $\left(\Delta m_{23}^{2} L / E_{\nu} \ll 1\right.$ for the relevant energies and lengths of travel); and it is smaller than the effective mass excess induced on electron neutrinos by matter effects, $\Delta m_{23}^{2} \ll A$, with $A$ as in Eq. (3). De facto, these conditions simply amount to $\Delta m_{23}^{2} \ll 10^{-4} \mathrm{eV}^{2}$. In practice we can set $\mu_{i}^{2}=0$ in the analysis of oscillations over terrestrial baselines.

For the mass pattern of Eq. (4), one of the three neutrino mixing angles and the $C P$-violating phase of the CKM matrix $U$ are unobservable. This is readily checked. We parametrize the CKM matrix in the unconventional order $U$ $\equiv U_{12} U_{13} U_{23}$, as follows:

$$
\begin{aligned}
& U \equiv U_{12} U_{13} U_{23} \equiv\left(\begin{array}{ccc}
c_{12} & s_{12} & 0 \\
-s_{12} & c_{12} & 0 \\
0 & 0 & 1
\end{array}\right)\left(\begin{array}{ccc}
c_{13} & 0 & s_{13} \\
0 & 1 & 0 \\
-s_{13} & 0 & c_{13}
\end{array}\right) \\
& \times\left(\begin{array}{ccc}
1 & 0 & 0 \\
0 & c_{23} & s_{23} e^{i \delta} \\
0 & -s_{23} e^{-i \delta} & c_{23}
\end{array}\right)
\end{aligned}
$$

with $s_{12}=\sin \left(\theta_{12}\right)$, etc. The vacuum mass matrix in flavor space $M_{F}^{v a c} \equiv U M_{K}^{v a c} U^{\dagger}$ does not depend on $s_{23}$, or on $\delta$. The mixing matrix is effectively reduced to $U \equiv U_{12} U_{13}$.

In the approximation we are discussing, $\mathrm{m}^{2}$ is the only relevant mass-scale difference and the vacuum transition probabilities between different neutrino flavors are

$$
\begin{aligned}
& P\left(\nu_{e} \rightarrow \nu_{\mu}\right)=4 s_{12}^{2} c_{12}^{2} c_{13}^{4} \sin ^{2}\left(\frac{m^{2} L}{4 E_{\nu}}\right), \\
& P\left(\nu_{e} \rightarrow \nu_{\tau}\right)=4 s_{13}^{2} c_{13}^{2} c_{12}^{2} \sin ^{2}\left(\frac{m^{2} L}{4 E_{\nu}}\right), \\
& P\left(\nu_{\mu} \rightarrow \nu_{\tau}\right)=4 s_{13}^{2} c_{13}^{2} s_{12}^{2} \sin ^{2}\left(\frac{m^{2} L}{4 E_{\nu}}\right) .
\end{aligned}
$$

The probabilities $P\left(\nu_{e} \rightarrow \nu_{\mu}\right)$ and $P\left(\nu_{e} \rightarrow \nu_{\tau}\right)$ are quadratically suppressed for small $s_{12}$ and $s_{13}$, while $P\left(\nu_{\mu} \rightarrow \nu_{\tau}\right)$ is quartically suppressed. The situation in matter, however, is drastically different.

It is convenient to work in the "kinetic" basis wherein $M_{K}^{v a c}$ is diagonal. The effect of matter is fully encrypted in a modification of the squared mass matrix:

$$
M_{K}^{m a t} \equiv M_{K}^{v a c}+U^{\dagger}\left(\begin{array}{ccc}
A+B & 0 & 0 \\
0 & B & 0 \\
0 & 0 & B
\end{array}\right) U,
$$

where, as is well known, $B$ arises from flavor-universal forward-scattering neutral current interactions while $A$, given by Eq. (3), arises from the charged-current contribution specific to $\bar{\nu}_{e} e$ and $\nu_{e} e$ scattering.

To illustrate how matter effects lift the vacuum degeneracy between two mass eigenstates, we diagonalize $M_{K}^{\text {mat }}$ to first order in $\mathrm{A} / \mathrm{m}^{2}$, temporarily assumed to be small (the exact formulas, used in the numerical results, are presented 
in Appendix A). To order zero in this expansion there are two equal eigenvalues, so that we must follow the usual rules of degenerate perturbation theory. Write $M_{K}^{m a t}=M^{[0]}$ $+M^{[1]}$ with

$$
\begin{aligned}
M^{[0]} & =\left(\begin{array}{ccc}
0 & 0 & 0 \\
0 & m^{2}+A s_{12}^{2} & A c_{12} s_{12} s_{13} \\
0 & A c_{12} s_{12} s_{13} & m^{2}+A c_{12}^{2} s_{13}^{2}
\end{array}\right), \\
M^{[1]} & =\left(\begin{array}{ccc}
A c_{12}^{2} c_{13}^{2} & A c_{12} c_{13} s_{12} & A c_{13} c_{12}^{2} s_{13} \\
A c_{12} c_{13} s_{12} & 0 & 0 \\
A c_{13} c_{12}^{2} s_{13} & 0 & 0
\end{array}\right),
\end{aligned}
$$

where we have subtracted the common entry $B$, which plays no role in neutrino mixing. We must diagonalize $M^{[0]}$ exactly to lift the degeneracy in $M^{v a c}$, then the second term can be consistently treated in perturbation theory.

To order $\mathrm{A} / \mathrm{m}^{2}$, the flavor and kinetic mass matrices, $M_{F}^{m a t}$ and $M_{K}^{m a t}$, are

$$
\begin{aligned}
& M_{F}^{m a t}=U_{12} U_{13} U_{m a t} M_{K}^{m a t} U_{m a t}^{\dagger} U_{13}^{\dagger} U_{12}^{\dagger}, \\
& M_{K}^{m a t}=\operatorname{Diag}\left[A c_{12}^{2} c_{13}^{2} ; m^{2} ; m^{2}+A\left(s_{12}^{2}+c_{12}^{2} s_{13}^{2}\right)\right],
\end{aligned}
$$

where

$$
U_{\text {mat }} \equiv\left(\begin{array}{ccc}
1 & 0 & 0 \\
0 & c_{23}^{\text {mat }} & s_{23}^{\text {mat }} \\
0 & -s_{23}^{\text {mat }} & c_{23}^{\text {mat }}
\end{array}\right) \text {, }
$$

and the sine of the mixing angle in matter is

$$
s_{23}^{m a t}=s_{12} / \sqrt{s_{12}^{2}+c_{12}^{2} s_{13}^{2}} .
$$

There are two important points to notice. First, instead of one mass difference as in vacuum, we have two:

$$
\begin{gathered}
\Delta m_{12}^{2} \sim m^{2}, \\
\Delta m_{23}^{2}=A\left(s_{12}^{2}+c_{12}^{2} s_{13}^{2}\right),
\end{gathered}
$$

one of $O\left(\mathrm{~m}^{2}\right)$, the other of $O(A)$, the matter-induced mass. Second, the matrix $U_{\text {mat }}$ plays the same role as the mixing matrix $U_{23}$ in the generic mixing scenario of three neutrinos. The effect of matter is simply to split the degenerate eigenvalues and induce the effective angle $s_{23}^{\text {mat }}$ of Eq. (12). The crucial point is that this angle can be large even if $s_{12}$ and $s_{13}$ are small. To this order in $A / \mathrm{m}^{2}$, the condition for maximal mixing is $s_{13}=s_{12} / c_{12}$; in a parametrization-independent language, this is equivalent to the requirement that the mixing between the second and third eigenstates with the electron flavor state be the same: $U(e 2)=U(e 3)$.

\section{B. Oscillation probabilities}

For the Earth's electron density appearing in A, Eq. (3), it is a good approximation [12] to consider a piecewiseconstant density profile: a negligible density for neutrinos traversing the atmosphere, $\rho_{m}=5 \mathrm{~g} / \mathrm{cm}^{3}$ for the mantle, and $\rho_{c}=11 \mathrm{~g} / \mathrm{cm}^{3}$ for a core of $3500 \mathrm{~km}$ radius. The matterinduced squared "mass" can then be expressed as $A$ $=2 \sqrt{2} G_{F} E_{\nu}\left\langle n_{e}\left(c_{\nu}\right)\right\rangle$, where the electron density is averaged over the neutrino trajectory, and $c_{\nu}$ is the cosine of its zenith angle. The oscillation probabilities depend on the two mass differences of Eq. (13) and have the same form as the $C P$-conserving part of the general three-flavor vacuumtransition probabilities:

$$
P_{\nu_{\alpha} \nu_{\beta}}\left(E_{\nu}, c_{\nu}\right)=-4 \sum_{k>j} \operatorname{Re}\left[W_{\alpha \beta}^{j k}\right] \sin ^{2}\left(\frac{\Delta m_{j k}^{2} L\left(c_{\nu}\right)}{4 E_{\nu}}\right),
$$

with $W_{\alpha \beta}^{j k} \equiv\left[U_{\alpha j} U_{\beta j}^{*} U_{\alpha k}^{*} U_{\beta k}\right]$, and $U \equiv U_{12} U_{13} U_{m a t}$. The distance $L\left(c_{\nu}\right)$ is

$$
L\left(c_{\nu}\right)=R_{\otimes}\left[\sqrt{\left(1+l / R_{\otimes}\right)^{2}-s_{\nu}^{2}}-c_{\nu}\right],
$$

where $R_{\otimes}$ is the radius of the Earth and $l \sim 15 \mathrm{~km}$ is the typical height at which primary cosmic rays interact in the atmosphere.

Consider the $\nu_{\mu} \leftrightarrow \nu_{\tau}$ entry of Eqs. (14):

$$
P_{\nu_{\mu} \nu_{\tau}}\left(E_{\nu}, c_{\nu}\right)=\sin ^{2}\left(2 \theta_{23}^{m a t}\right) \sin ^{2}\left(\frac{\Delta m_{23}^{2} L\left(c_{\nu}\right)}{4 E_{\nu}}\right)+O\left(s_{12}^{2}, s_{13}^{2}\right) .
$$

Even if $s_{12}$ and $s_{13}$ are small, $P_{\nu_{\mu} \nu_{\tau}}$ can be maximal, since $\theta_{23}^{m a t} \simeq \pi / 4$ for $s_{12} \simeq s_{13}$. In the limit $s_{12}, s_{13} \rightarrow 0, \Delta m_{23}^{2} \rightarrow 0$ and the oscillation probability vanishes: there cannot be oscillations if all CKM angles are zero.

Large $\nu_{\mu} \rightarrow \nu_{\tau}$ oscillations take place for $s_{12}, s_{13}$ small, but bounded from below by the condition $\Delta m_{23}^{2} R_{\otimes} / E_{\nu}$ $\sim O(1)$. For this parameter range one should still check the size of $\nu_{e} \rightarrow \nu_{\mu}, \nu_{\tau}$ transitions, which are not observed. This turns out not to be a problem for the atmospheric anomaly, because the $\nu_{\mu} / \nu_{e}$ flux ratio is close to 2 , and in the region of maximal mixing and small vacuum angles $P\left(\nu_{e} \rightarrow \nu_{\mu}\right)$ $\sim P\left(\nu_{e} \rightarrow \nu_{\tau}\right)$. Consequently, the number of disappearing $\nu_{e}$ can be compensated by the number of $\nu_{\mu}$ s that oscillate into $\nu_{e} \mathrm{~s}$ [13]. But a large $\nu_{e}$ disappearance probability can lead to a violation of the stringent bounds imposed by the $\mathrm{CHOOZ}$ experiment $[14,15]$. We shall see that $\mathrm{CHOOZ}$, but not SK, disfavors our small-angle scenario.

\section{Relation to the conventional scenario}

The degenerate-neutrino scenario involves only one vacuum mass difference in the description of terrestrial experiments. This is also the case in the scenarios considered in most previous analyses of atmospheric data, even with three families [5], but with a single dominant mass difference.

As it turns out, the degenerate scenario is exactly equivalent to the conventional one of [5] with $\bar{\Delta} m_{23}^{2}=-m^{2}$ (in vacuum oscillations the sign of this difference would be unobservable). To see this equivalence explicitly, it suffices to 
consider their vacuum CKM matrix, which is written in the customary order $\bar{U} \equiv \bar{U}_{23}\left(\bar{s}_{23}\right) \bar{U}_{13}\left(\bar{s}_{13}\right)$, and to obtain from it the matrix of Eq. (5) via the substitutions:

$$
\begin{aligned}
& \bar{s}_{23}^{2}=\frac{s_{12}^{2} c_{13}^{2}}{s_{12}^{2}+s_{13}^{2} c_{12}^{2}}, \\
& \bar{s}_{13}^{2}=c_{12}^{2} c_{13}^{2} .
\end{aligned}
$$

Note that small mixing angles in the degenerate parametrization may correspond to large mixings in the conventional one. In particular, a region of arbitrarily small mixing angles $s_{12}^{2}, s_{13}^{2}$ of our mass-degenerate scenario is mapped to a domain around the values $\bar{s}_{23}^{2} \sim 1 / 2$ and $\bar{s}_{13}^{2} \sim 1$ of the conventional parametrization. The most "natural" parametrizations are the ones in which the rotation matrices $U_{i j}$ act on the mass eigenstates in order of decreasing degeneracy. The conventional parametrization, used in the Particle Data Group book [4], is natural for the quark sector with its hierarchical mass splitting, but not necessarily for the lepton sector. The parametrization we use, Eq. (5), is natural for the partially degenerate mass pattern that we are considering [16].

As we saw in the previous subsection, the presence of degenerate eigenstates in vacuum can lead to large transition probabilities in matter. The enhacement of transition probabilities in matter in the context of three-family mixing with two degenerate neutrinos has been discussed before in [11] and, in the context of the three-maximal mixing model, in [13]. The parametrization we use here clarifies the origin and generality of the effect.

\section{ZENITH ANGLE AND ENERGY DISTRIBUTION OF THE SK EVENTS}

The data of the SK collaboration, as well as their Monte Carlo expectations for the case in which there are no neutrino oscillations, are binned in the azimuthal angle of the observed electrons and muons, and in their energy (in the case of muons the level of containment within the detector also distinguishes different data samples). To reproduce these results one must convolute neutrino fluxes and survival probabilities with charged-current differential cross sections and implement various efficiencies and cuts. This being an elaborate procedure, in Appendix D we check our results by reproducing the no-oscillation Monte Carlo results of SK, as well as the neutrino "parent energy" spectrum: the azimuthally averaged neutrino flux weighted with the integrated neutrino cross section and with the efficiencies of the various data samples.

In the rest of this section we review how the data are binned, we specify our procedure, and we analyze the fits to the conventional oscillation scenario, as well as to our massdegenerate alternative.

\section{A. The data samples}

The SK collaboration chooses to bin the observed charged-lepton energies in a few samples. The electron can- didates are subdivided into sub-GeV (sgev) and multi-GeV (mgev). The muon candidates are distinguished as sgev and mgev, partially and fully contained (PC, FC), and throughgoing (thru). To set apart these categories, we introduce selection functions $\operatorname{Th}_{s, l}\left(E_{l}, c_{l}\right)$, with $s=$ sgev, mgev and $l$ $=e, \mu$, that depend on the energy $E_{l}$ and on the cosine $c_{l}$ of the azimuthal angle of the outgoing lepton $\left(c_{l}=1\right.$ is vertically down-going). In computing the number of events, these selection functions will weight the product of neutrino flux and cross section. For the sgev events,

$$
\operatorname{Th}_{\mathrm{sgev}, l}\left(E_{l}\right) \equiv \Theta\left[E_{\mathrm{th}, l}-E_{l}\right] \Theta\left[E_{l}-E_{\mathrm{min}, 1}\right]
$$

with $E_{\text {th }, e(\mu)}=1.33$ (1.4) $\mathrm{GeV}, E_{\min , e(\mu)}=100$ (200) $\mathrm{MeV}$ for $e(\mu)$, respectively. For the mgev electron events, $\operatorname{Th}_{\mathrm{mgev}, e}\left(E_{l}\right) \equiv \Theta\left[E_{l}-E_{\mathrm{th}, e}\right]$.

For the mgev muons, we must distinguish between partially and fully contained events:

$$
\begin{aligned}
& \operatorname{Th}_{\text {mgev-PC }, \mu}\left(E_{\mu}, c_{\mu}\right) \equiv \Theta\left[E_{\mu}-E_{\text {th }, \mu}\right] \operatorname{PC}\left(E_{\mu}, c_{\mu}\right), \\
& \operatorname{Th}_{\text {mgev-FC }, \mu}\left(E_{\mu}, c_{\mu}\right) \equiv \Theta\left[E_{\mu}-E_{\text {th }, \mu}\right] \operatorname{FC}\left(E_{\mu}, c_{\mu}\right),
\end{aligned}
$$

where the functions $\mathrm{FC}\left(E_{\mu}, c_{\mu}\right), \quad \operatorname{PC}\left(E_{\mu}, c_{\mu}\right)$ measure the fraction of the total fiducial volume in which a neutrino interaction could produce a $\mu$ with energy $E_{\mu}$ and zenith direction $c_{\mu}$ that either stops before exiting the detector (FC) or escapes (PC). We have explicitly constructed $\mathrm{FC}\left(E_{\mu}, c_{\mu}\right)$ and $\mathrm{PC}\left(E_{\mu}, c_{\mu}\right)$ using the shape and size of the detector and the $\mu$ range in water, $R_{w}\left(E_{\mu}\right)$, as a function of energy, and describe this in Appendix $\mathrm{C}$.

We have also devised a through-going muon selection function $\operatorname{Th}_{\text {thru, } \mu}\left(E_{\mu}, c_{\mu}\right)$. The effective target mass of the rock surrounding SK depends on energy via the muon range in water and in rock, $R_{r}\left(E_{\mu}\right)$. The observed muon energy is required to be greater than $E_{\min }^{\prime}=1.6 \mathrm{GeV}$, implying that its trajectory must be longer than $7 \mathrm{~m}$. The function $\mathrm{Th}_{\mathrm{thru}, \mu}$ must account for the detector's effective area for such tracks, $\mathrm{A}\left(E_{\mu}, c_{\mu}\right)$, which depends, via the muon range, on the muon energy as it enters the detector, and on its zenith angle. Furthermore, the selection function for through-going muons must take into account that their flux, as given by SK, is defined as the number of events divided by the effective area for a muon of energy $E_{\min }^{\prime}[17]$. All in all,

$$
\mathrm{Th}_{\mathrm{thru}, \mu}\left(E_{\mu}, c_{\mu}\right) \equiv\left[R_{r}\left(E_{\mu}\right)-R_{r}\left(E_{\text {min }}^{\prime}\right)\right] \frac{\mathrm{A}\left(E_{\mu}, c_{\mu}\right)}{\mathrm{A}\left(E_{\text {min }}^{\prime}, c_{\mu}\right)} \text {. }
$$

This effective area is given in Appendix C.

\section{B. Number of events}

Let $d \Phi_{\nu}\left(E_{\nu}, c_{\nu}\right) / d E_{\nu} d c_{\nu}$ and $d \bar{\Phi}_{\nu}\left(E_{\nu}, c_{\nu}\right) / d E_{\nu} d c_{\nu}$ be the atmospheric neutrino fluxes of $\nu=\nu_{e}, \nu_{\mu}$ and their antiparticles, with $E_{\nu}$ being the neutrino energy and $c_{\nu}$ its zenith angle (we use the Bartol code [18] of atmospheric neutrino fluxes at the Kamiokande site). Let $d \sigma\left(E_{\nu}, E_{l}, c_{\beta}\right) / d E_{l} d c_{\beta}$ and $d \bar{\sigma}\left(E_{\nu}, E_{l}, c_{\beta}\right) / d E_{l} d c_{\beta}$ be the neutrino and antineutrino 
charged-current cross sections, which depend on $E_{\nu}$, on the outgoing lepton energy $E_{l}$, and on the cosine of the scattering angle between the two particles, $c_{\beta}$. In Appendix B, we discuss in detail the cross sections used in our analysis. The zenith angle of the outgoing lepton $c_{l}$, which is the measured quantity, is a function of $c_{\nu}, c_{\beta}$, and of the azimuthal angle, $\phi$, of the outcoming lepton in the target rest frame.

Let $N_{s, l}^{0}(c), N_{s, l}^{\text {osc }}(c)$ be the expected number of chargedcurrent events in the sample $s$ ( $s=$ sgev, mgev-pc, mgev-fc, thru) for $l=e, \mu$ and in the bin in the zenith cosine with central value $c$, for the no-oscillation (0) and oscillation (osc) hypotheses. For the sgev and mgev samples, the theoretical prediction is given by

$$
\begin{aligned}
N_{s, l}^{\mathrm{osc}}(c)= & K_{s, l} \int d E_{\nu} d c_{\nu} d E_{l} d c_{\beta} d \phi \mathrm{Th}_{\mathrm{s}}\left(E_{l}, c_{l}\right) \\
& \times\left(\Theta\left[c_{l}-c+\delta\right]-\Theta\left[c_{l}-c-\delta\right]\right) \\
& \times \sum_{\nu^{\prime}=\nu_{e}, \nu_{\mu}}\left[\sigma\left(E_{\nu}, E_{l}, c_{\beta}\right) \frac{d \Phi_{\nu^{\prime}}}{d E_{\nu} c_{\nu}} P_{\nu^{\prime} \nu}\left(E_{\nu}, c_{\nu}\right)\right. \\
& \left.+\bar{\sigma}\left(E_{\nu}, E_{l}, c_{\beta}\right) \frac{d \Phi_{\nu^{\prime}}}{d E_{\nu} d c_{\nu}} \bar{P}_{\nu^{\prime} \nu}\left(E_{\nu}, c_{\nu}\right)\right]
\end{aligned}
$$

for the oscillation hypothesis, with $P_{\nu^{\prime} \nu}\left(E_{\nu}, c_{\nu}\right)$ and $\bar{P}_{\nu^{\prime} \nu}\left(E_{\nu}, c_{\nu}\right)$ being the oscillation probabilities from flavor $\nu^{\prime}$ to flavor $\nu$ for neutrinos and antineutrinos, respectively. To obtain $N^{0}$, take $P_{\nu^{\prime} \nu}=\bar{P}_{\nu^{\prime} \nu}=\delta_{\nu^{\prime} \nu}$. The $\Theta$ functions in Eq. (21) express the constraint that $c_{l}$ be in the bin with central value $c$ and width $2 \delta=0.4$, the binning used in SK for the sgev and mgev samples. Finally, $K_{s, l}$ are normalization constants, which ensure that the total number of events in each sample is the same as in the SK Monte Carlo data for the nonoscillation hypothesis. By choosing these factors by hand, we skirt the question of efficiencies for electron or muon detection and for single- or multiple-ring events: all we need to assume is that they are roughly constant within a given data sample, which we believe to be the case. We neglect the cross-talk between different samples. An important difference with the analysis of Ref. [7] is that we do not use the unpublished neutrino parent energies obtained by SK for the different samples, but we construct our own.

For the flux of through-going muons, we have

$$
\begin{aligned}
\Phi_{\mathrm{thru}}^{\mathrm{osc}}(c)= & K_{\mathrm{thru}} \int d \phi d c_{\beta} d E_{\mu} d c_{\nu} d E_{\nu} \operatorname{Th}_{\mathrm{thru}, \mu}\left(E_{\mu}, c_{\mu}\right) \\
& \times\left(\Theta\left[c_{\mu}-c+\delta\right]-\Theta\left[c_{\mu}-c-\delta\right]\right) \\
& \times \sum_{\nu^{\prime}=\nu_{e}, \nu_{\mu}}\left[\sigma\left(E_{\nu}, E_{\mu}, c_{\beta}\right) \frac{d \Phi_{\nu^{\prime}}}{d E_{\nu} d c_{\nu}} P_{\nu^{\prime} \nu_{\mu}}\left(E_{\nu}, c_{\nu}\right)\right. \\
& \left.+\bar{\sigma}\left(E_{\nu}, E_{\mu}, c_{\beta}\right) \frac{d \Phi_{\nu^{\prime}}}{d E_{\nu} d c_{\nu}} \bar{P}_{\nu^{\prime} \nu_{\mu}}\left(E_{\nu}, c_{\nu}\right)\right]
\end{aligned}
$$
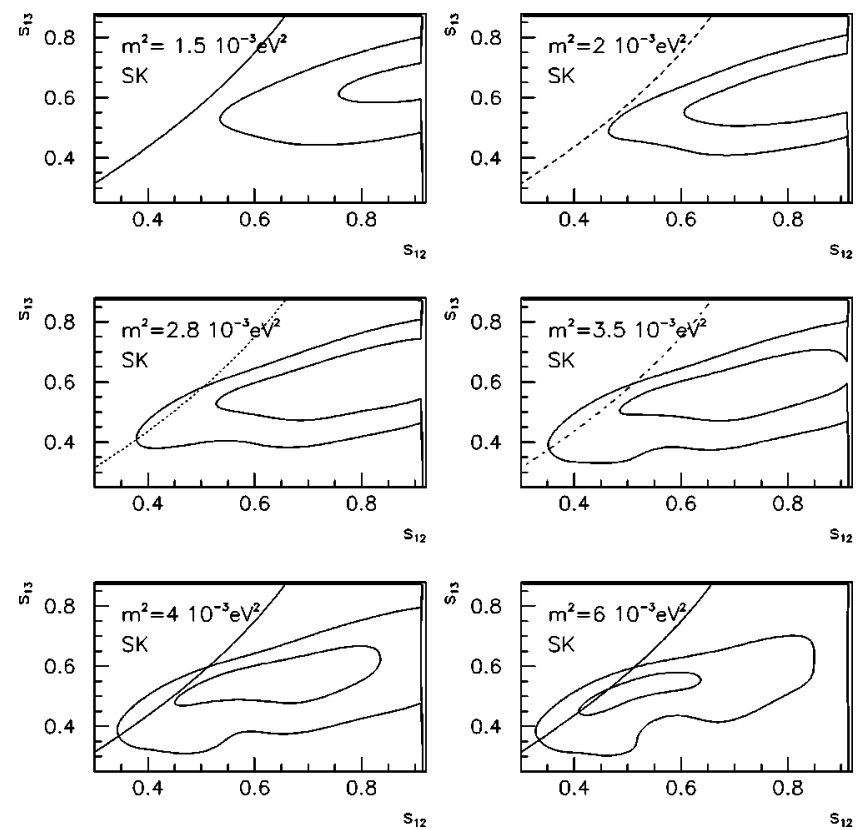

FIG. 1. 68.5,99\% C.L. intervals allowed by SK data alone, for different values of $\mathrm{m}^{2}$.

for the case with oscillations. The width of the zenith angle bins in this sample is $2 \delta=0.1$.

\section{Results of the analysis of the SK data}

We have performed a $\chi^{2}$ analysis of the oscillation hypothesis in our mass-degenerate scenario, for both signs of $\mathrm{m}^{2}$, using the full 52 kiloton-year data sample gathered by the Super-Kamiokande collaboration in 848 days of exposure [19]. The case of negative $m^{2}$ is exactly equivalent to the conventional scenario considered in [7], as shown in Sec. II. The measured quantities are the 30 zenith angle bins measured by SK in the five types of data samples. The choice of an error correlation matrix is nontrivial, as there are large theoretical uncertainties in the input neutrino flux, which induce large correlations between the errors in the different measured quantities. We have constructed the error correlation matrix in the same way as the authors of [7], to whom we refer for details. To gauge the incidence of these "systematic" errors on the results, we have also performed the analysis with only statistical uncertainties.

In Fig. 1 we show, in the plane $s_{12}-s_{13}$ and for several positive values of $\mathrm{m}^{2}$, the contour lines delimiting the allowed regions at 68.5 and $99 \%$ confidence. In Fig. 2 the same information is displayed for negative $m^{2}$. The region of maximal mixing in the conventional parametrization corresponds to the values $s_{12} \sim 1$ and $s_{13} \sim 1 / \sqrt{2}$ of our parametrization. This region is favored for the smaller allowed values of $m^{2}$ : the top two rows of Fig. 1. At the larger values of $m^{2}$, however, the contours extend largely to a region with significantly smaller vacuum angles, the oscillation probabilities being enhanced by matter effects. We draw for comparison the line corresponding to maximal mixing in the perturbative approximation of Eq. (12), valid for the larger $m^{2}$ values. The allowed regions at small angles are close to this line, as 

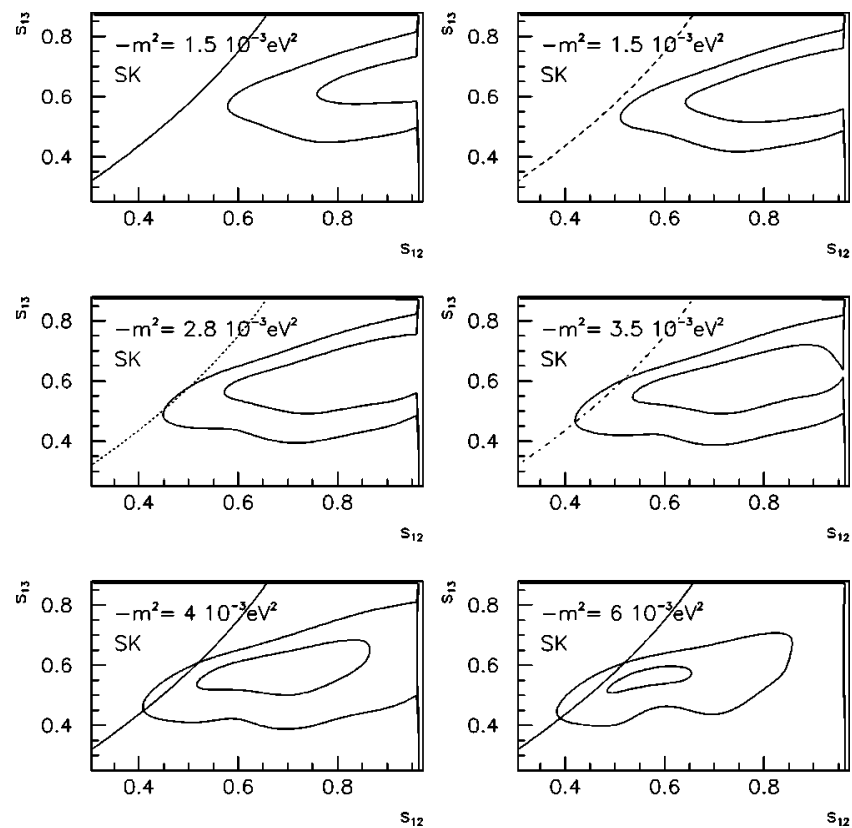

FIG. 2. The same as Fig. 1, but for negative $m^{2}$.

expected. At values of $m^{2}$ smaller than those shown in the figure, the allowed regions shrink around the conventional maximal-mixing solution.

The minimum $\chi^{2}$ is obtained for $\left|m^{2}\right| \sim 3.5 \times 10^{-3} \mathrm{eV}^{2}$, independently of whether the errors are taken to be purely statistical, or estimates of flux uncertainties are also included. This result is in good agreement with that found by the SK collaboration in a two-family mixing context. We do not find such an agreement on the optimized mixing angles. The best-fit angles in our parametrization are $s_{12}^{2}$ $\sim 0.42(0.45)$ and $s_{13}^{2} \sim 0.31(0.33)$ for positive (negative) $\mathrm{m}^{2}$, which in the conventional parametrization correspond to $\bar{s}_{23}^{2}=0.48(0.48)$ and $\bar{s}_{13}^{2}=0.4(0.37)$, not so far from the so-called trimaximal mixing model $\left(s_{12}^{2}=1 / 2, s_{13}^{2}=1 / 3\right)$ [13].

As one can see in Fig. 1, for the optimal $m^{2}=3.5$ $\times 10^{-3} \mathrm{eV}^{2}$, the values $s_{12} \sim s_{13} \sim 0.5$ are allowed at the $1 \sigma$ confidence, corresponding to $U_{e 1}=c_{12} c_{13} \sim 0.75$. This means that with a $\nu_{e}$ that is dominantly the light $\nu_{1}$ mass eigenstate, we obtain a fit as good as the conventional maximal mixing one. This is the explicit quantification of our claim in the abstract: an effectively maximal mixing can be induced by matter effects if $\nu_{e}$ 's contain comparable admixtures of the heavier eigenstates, even if they are not large. The $\mathrm{CHOOZ}$ data, however, disfavor these relatively large- $m^{2}$ and smallangle solutions.

\section{CHOOZ CONSTRAINTS}

The reactor experiment $\mathrm{CHOOZ}$ provides tight upper limits on the $\bar{\nu}_{e}$ disappearance probability in a domain with $\Delta m^{2}$ $\geqslant 10^{-3} \mathrm{eV}^{2}[14,15]$. This entails very strong strictures on $P_{\bar{\nu}_{e}} \bar{\nu}_{\mu}$ and $P_{\bar{\nu}_{e}} \bar{\nu}_{\tau}$ at atmospheric distances.

We have constrained our analysis of atmospheric data to
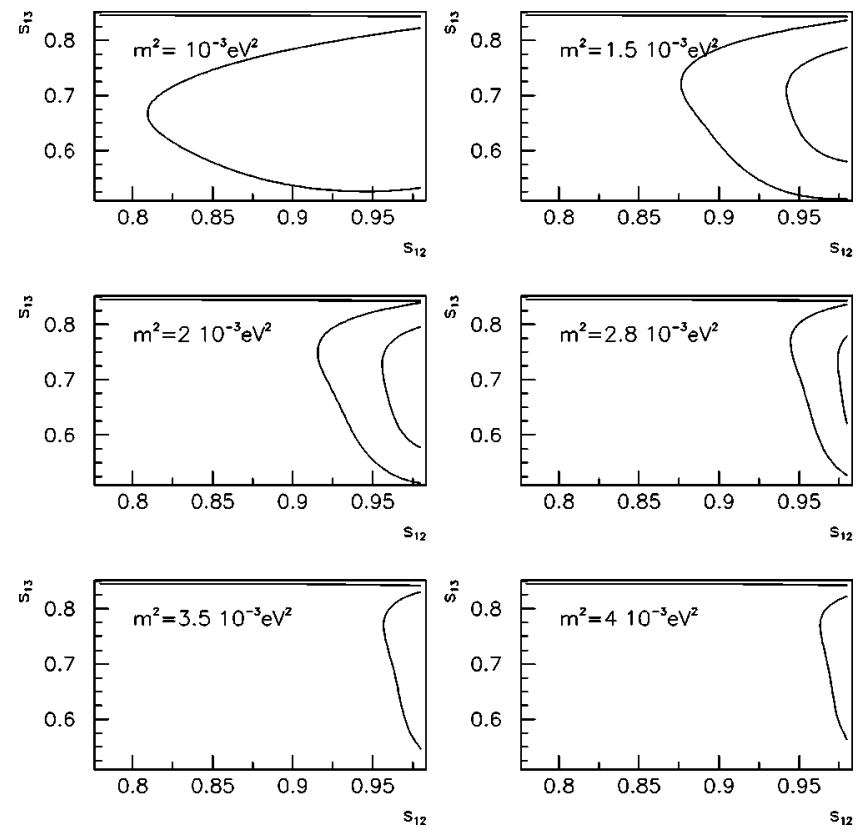

FIG. 3. $68.5,99 \%$ C.L. intervals allowed by SK and $\mathrm{CHOOZ}$ data for different values of $\mathrm{m}^{2}$.

comply with the CHOOZ results on the ratio $R$ of observed $e^{+}$ events to the number expected in the absence of oscillations:

$$
R=\frac{\int d E_{\nu} \Phi\left(E_{\nu}\right) \sigma\left(E_{\nu}\right) P_{\bar{\nu}_{e} \bar{\nu}_{e}}\left(E_{\nu}\right)}{\int d E_{\nu} \Phi\left(E_{\nu}\right) \sigma\left(E_{\nu}\right)}
$$

where $\Phi\left(E_{\nu}\right)$ is the spectrum of neutrinos, obtained by combining, in the appropriate proportions [15], the decay spectra of the different isotopes in the CHOOZ reactors [20]. In writing Eq. (23) we have approximated the efficiency as a constant, for lack of better information. The cross section, $\sigma\left(E_{\nu}\right)$, including the threshold effects, has been obtained from [21]. For the transition probabilities we can use Eq. (6), since matter effects are completely negligible.

The results of this combined SK-CHOOZ analysis are shown in Fig. 3 for positive $m^{2}$. The results for negative $m^{2}$ shown in Fig. 4 are very similar. Clearly the $\mathrm{CHOOZ}$ data favor the conventional maximal-mixing solution as the only acceptable one.

In Fig. 5, we show the minimum $\chi^{2}$ as a function of mass, for positive $m^{2}$. On the left we include theoretical flux errors as in [7], while on the right only statistical uncertainties are taken into account. Reassuringly, the theoretical errors have a small incidence on the results. In Fig. 6, we show the results for negative $m^{2}$. For both signs of the mass difference, the minimum of the $\chi^{2}$ occurs at $\left|m^{2}\right|=2-2.5$ $\times 10^{-3} \mathrm{eV}^{2}$, which is slightly smaller than the value obtained in the combined analysis of [7] $\left(\left|\mathrm{m}^{2}\right| \sim 2.8\right.$ $\times 10^{-3} \mathrm{eV}^{2}$ ). Concerning the mixing amplitudes, we find as the best fit for the important $[22,16]$ angle $\bar{\theta}_{13}$-in the conventional parametrization-at $\bar{\theta}_{13}=6^{\circ}$, to be compared to $8^{\circ}$ 

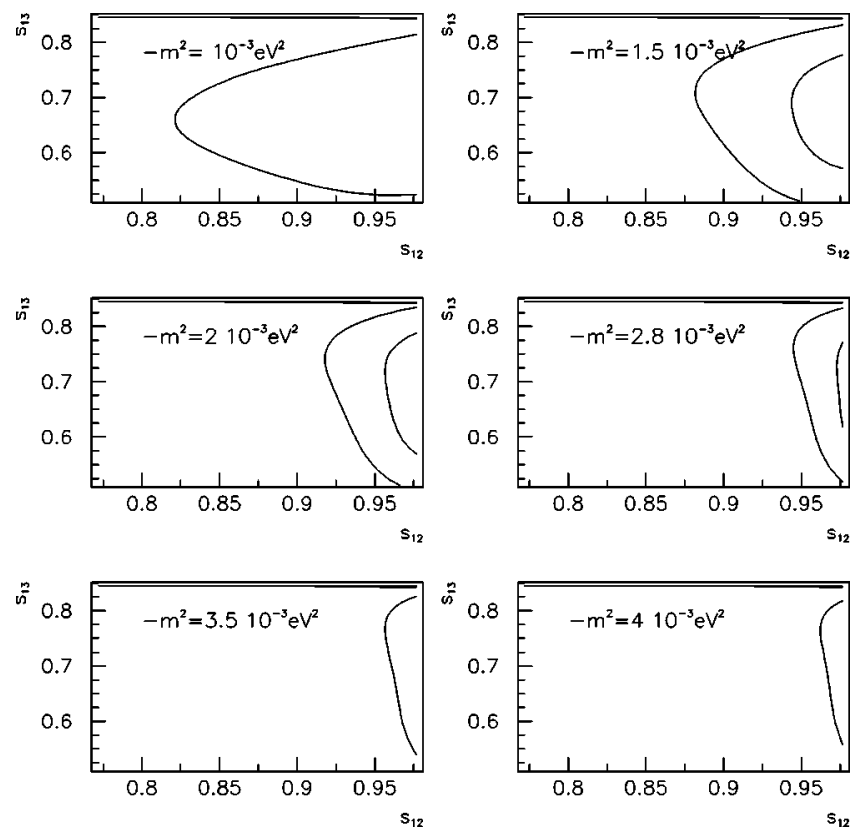

FIG. 4. The same as Fig. 3, but for negative $m^{2}$.

found in [7]. However, the $\chi^{2}$ curve is flat enough for $\bar{\theta}_{13}$ $=0$ to be perfectly compatible with the data.

In Figs. 7 and 8 we show the impressive agreement between the SK zenith angle distributions and our best-fit oscillation hypothesis, obtained including the $\mathrm{CHOOZ}$ constraint.

Incidentally, for the trimaximal mixing model we get $\chi^{2}$ $=42$ (44) (for 30 degrees of freedom: 31 data minus one
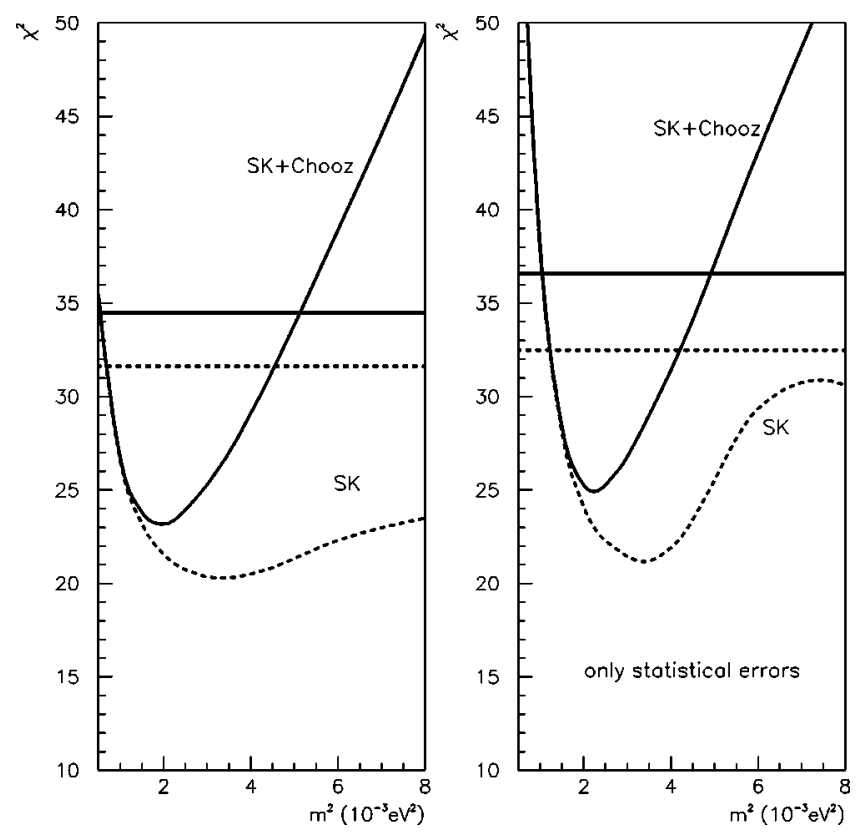

FIG. 5. Minimum $\chi^{2}$ as a function of $m^{2}$. The solid line corresponds to $\mathrm{SK}+\mathrm{CHOOZ}$ data, while the dashed line includes only SK data. The curves on the left plot include theoretical uncertainties as in [7], while the one on the right includes only statistical errors. The horizontal lines correspond to $99 \%$ C.L. intervals for three degrees of freedom.
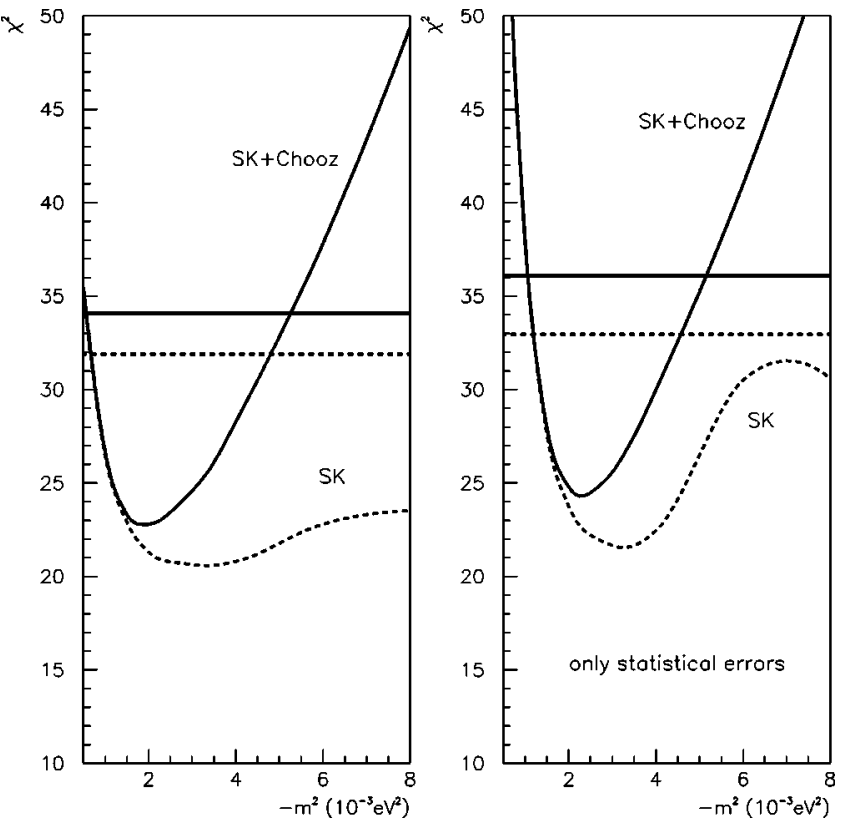

FIG. 6. The same as Fig. 5 but for negative $m^{2}$.

free parameter $\mathrm{m}^{2}$ ) for positive (negative) $\mathrm{m}^{2}$ at $\left|\mathrm{m}^{2}\right|$ $=10^{-3} \mathrm{eV}^{2}$, a mass value for which the CHOOZ constraint is inoperative. The $\chi^{2}$ rises rapidly for larger $\left|m^{2}\right|$. The probability that this model is correct is below $10 \%$.

\section{CONCLUSIONS}

The neutrino squared mass difference used to explain the atmospheric neutrino anomaly in terms of oscillations is of
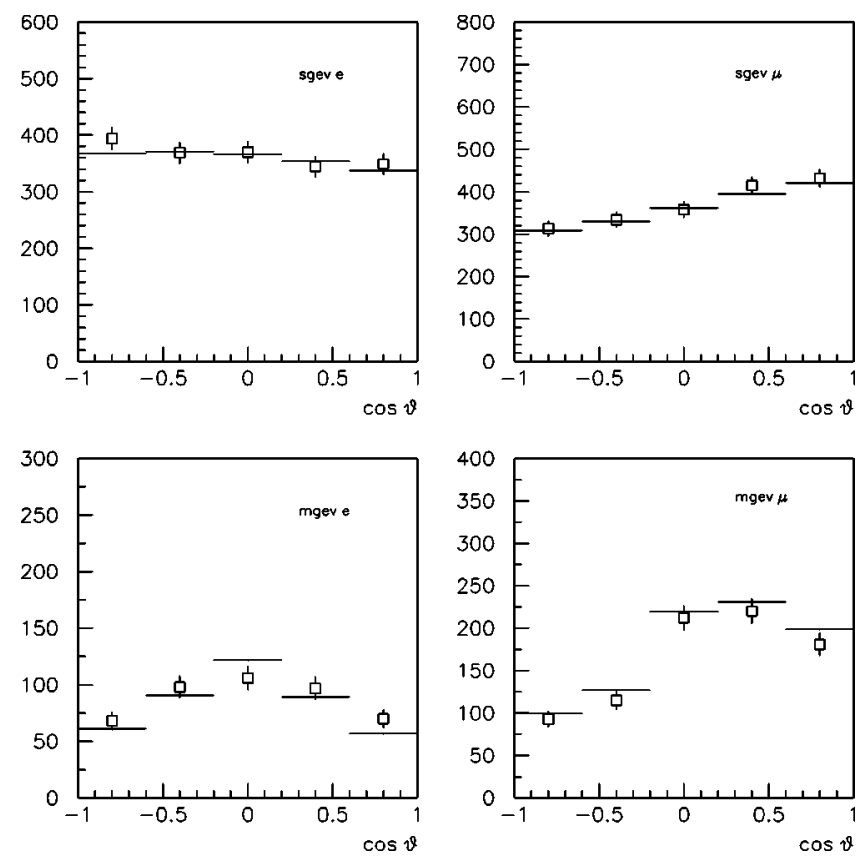

FIG. 7. Zenith angle distributions of $e$ and $\mu$ SuperKamiokande samples (squares) compared with the best-fit oscillation hypothesis: $m^{2}=2 \times 10^{-3} \mathrm{eV}^{2}$ and close to maximal mixing. The errors shown are only statistical. 


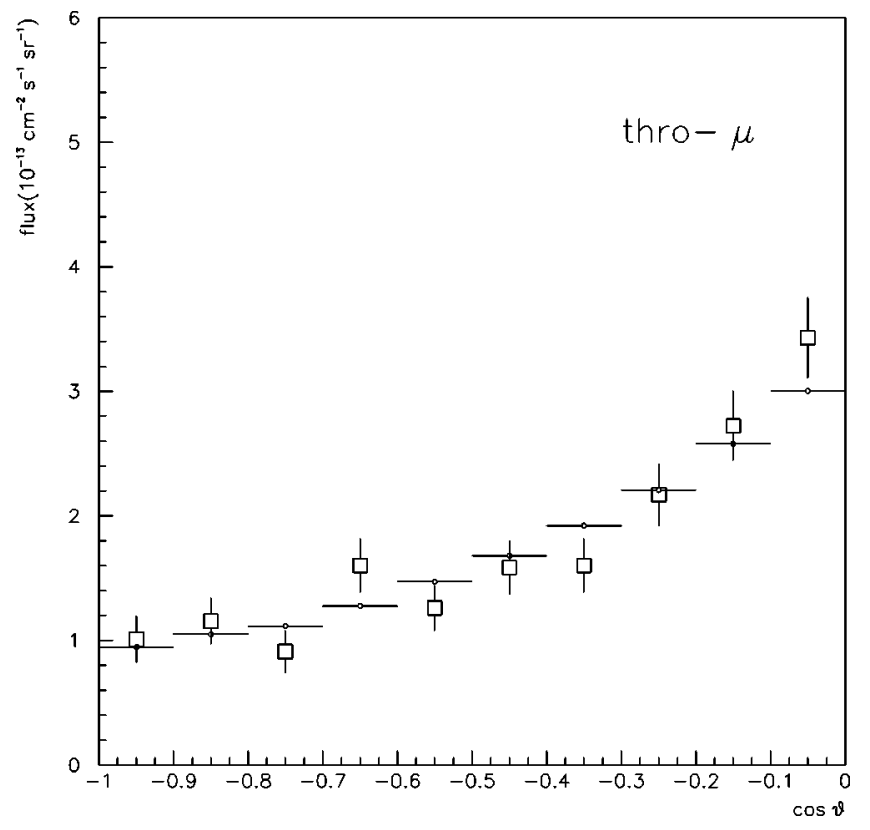

FIG. 8. Zenith angle distribution of through-going muons (squares) compared with the best fit oscillation hypothesis: $m^{2}=2$ $\times 10^{-3} \mathrm{eV}^{2}$ and close to maximal mixing.

the same order of magnitude as that induced by Earth matter effects, for a typical atmospheric neutrino energy. Triggered by this coincidence, we set out to study-in a scheme with three neutrinos and in minute detail-whether or not the large depletion of muon neutrinos observed by SK could be due, not to $a b$ initio large mixing angles, but to matterenhanced smaller-angle mixings. Our suspicion turned out to be correct: the SK data can be very satisfactorily explained with mixing angles that are far from maximal. But the constraints from $\mathrm{CHOOZ}$ on the survival of electron antineutrinos disfavor our nonmaximal solution. The conclusion that vacuum $\nu_{\mu} \leftrightarrow \nu_{\tau}$ neutrino transition probabilities are nearly maximal may be surprising, but it is here to stay.

Note added. After the completion of this work, a threefamily analysis was made public by the SuperKamiokande Collaboration at various conferences [32].

\section{ACKNOWLEDGMENTS}

We have had useful conversations with A. Donini, J. Ellis, F. Feruglio, G.L. Fogli, J. Gómez-Cadenas, M.C. Gonzalez-García, Y. Hayato, E. Lisi, M. Lusignoli, S. Rigolin, and O. Yasuda. We are indebted to the Bartol and Honda groups for providing us with their atmospheric neutrino fluxes. We warmly thank K. Scholberg for many illuminating discussions on the SK data and its interpretation. After the completion of this work, E. Lisi kindly pointed out to us Refs. [11], where matter effects in three-family mixing were previously discussed, and O. Peres pointed out a missprint in Eq. (8). The work of M.B.G. was also partially supported by CICYT project AEN/97/1678.

\section{APPENDIX A: OSCILLATION PARAMETERS}

The exact diagonalization of the mixing matrix in Eq. (7) results in the effective eigenmass differences:

$$
\begin{aligned}
& \Delta m_{12}^{2}=\frac{1}{2}\left(m^{2}-A+\sqrt{\left(m^{2}+A\right)^{2}-4 A m^{2} c_{13}^{2} c_{12}^{2}}\right), \\
& \Delta m_{23}^{2}=\frac{1}{2}\left(-m^{2}+A+\sqrt{\left(m^{2}+A\right)^{2}-4 A m^{2} c_{13}^{2} c_{12}^{2}}\right),
\end{aligned}
$$

and in a mixing matrix:

$$
U_{\text {mat }}=\left(\begin{array}{ccc}
-\left(c_{12} c_{13}-a_{+}\right) / b_{+} & 0 & \left(c_{12} c_{13}-a_{-}\right) / b_{-} \\
-s_{12} / b_{+} & s_{13} c_{12} / \sqrt{s_{12}^{2}+s_{13}^{2} c_{12}^{2}} & s_{12} / b_{-} \\
-s_{13} c_{12} / b_{+} & -s_{12} / \sqrt{s_{12}^{2}+s_{13}^{2} c_{12}^{2}} & s_{13} c_{12} / b_{-}
\end{array}\right),
$$

where

$$
\begin{gathered}
a_{ \pm} \equiv\left(A+m^{2} \pm \sqrt{\left(m^{2}+A\right)^{2}-4 A m^{2} c_{13}^{2} c_{12}^{2}}\right) /\left(2 A c_{12} c_{13}\right), \\
b_{ \pm} \equiv \sqrt{1+a_{ \pm}^{2}-2 a_{ \pm} c_{13} c_{12}} .
\end{gathered}
$$

\section{APPENDIX B: NEUTRINO CROSS SECTIONS}

The proper treatment of neutrino cross sections in water and rock, at energies ranging from $100 \mathrm{MeV}$ to hundreds of $\mathrm{GeV}$, is an arduous art. We do not attempt an elegant and complete analysis. Instead, we use a treatment thatnotwithsanding its oversimplifications-is capable of reproducing to an adequate level the observed total cross sections and scattering angle distributions, which are the ingredients needed for the data analysis.

We simplify the neutrino cross sections in water by considering only oxygen as a target, an isoscalar nucleus for which we ignore shadowing, but not Fermi-motion effects, which we treat as in [23]. We also neglect the muon mass. As the SK experimenters do, we build a cross section out of three dominant contributions: quasielastic $\left(\nu_{l} \mathcal{N} \rightarrow l \mathcal{N}^{\prime}\right)$, resonant one-pion production, and "deep" inelastic. In so doing we ignore the small contribution of the "diffractive", domain of relatively high energy, low $Q^{2}$.

For the quasielastic cross section we use the standard expression reviewed in [23], with $M_{A}=1.0 \mathrm{GeV}$ for the mass describing the axial form factor. For one-pion production we 


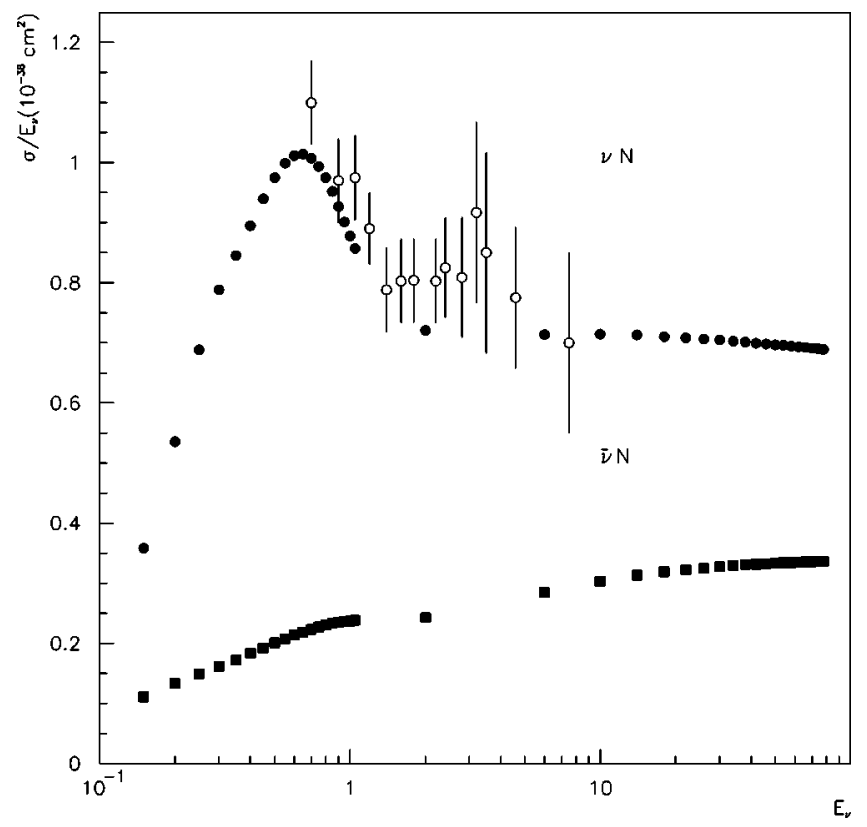

FIG. 9. Total cross section over neutrino energy for $\nu$ and $\bar{\nu}$ charged-current scattering on an isoscalar target.

use Eq. (22) of [24] for the excitation of the $N^{*}$ (1236) resonance of spin and isospin $3 / 2$. We assume that these contributions saturate the cross section for an invariant mass of the final hadrons $W \leqslant 1.4 \mathrm{GeV}$. Above that value we use a deepinelastic cross section with an exact Callan-Treiman constraint $F_{2}=2 x F_{1}$. For the structure functions $F_{2}$ and $F_{3}$ we use the compilation of [25]. As an excuse to extend this deep inelastic cross section to values of $Q^{2}$ as low as $0.4 \mathrm{GeV}^{2}$, we use $\xi$ scaling. This is known to deal correctly with the higher-twist target-mass corrections [26] and to interpolate

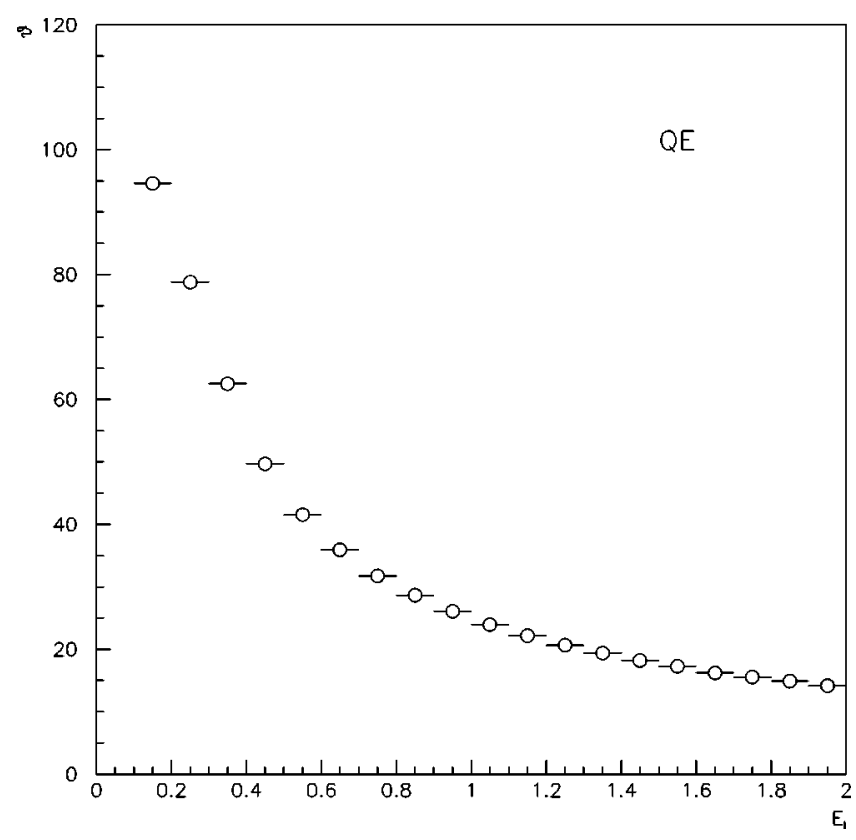

FIG. 10. Average scattering angle between the parent neutrino and the lepton for quasielastic events.
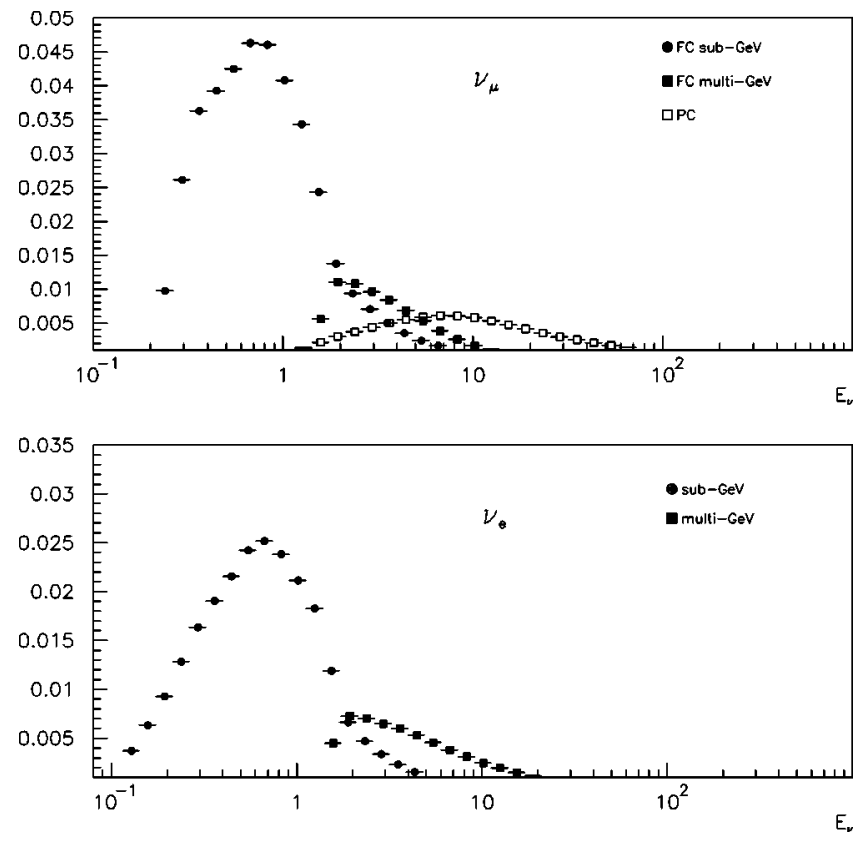

FIG. 11. Parent $\nu$ energies for $\mu$-like (sgev, FC-mgev, PCmgev) and $e$-like (sgev and mgev) events.

the resonant contributions in the sense of local duality [27]. Alas, the structure functions are not extracted from the data using $\xi$ scaling. But the authors of [28] have shown that, at least in the case of electroproduction, a blind a posteriori use of $\xi$ scaling improves the fit to the lower $Q^{2}$ data (their prescription consists in using the Bjorken-scaling crosssection expressions [4] and the structure functions extracted at higher $Q^{2}$, with the simple and not fully consistent $a$ posteriori substitution $x \rightarrow \xi$ in the argument of the structure functions).

In Fig. 9, we plot $\sigma_{\text {тот }}\left(E_{\nu}\right)$ as a function of $E_{\nu}$ for neutrinos and antineutrinos. The curves are a bit below the available data at low energies, no doubt reflecting the absence of a calculated diffractive contribution. The SK collaborators, as well as many other authors, extend the structure functions down to $Q^{2}=0$, thereby obtaining a slightly better "ffit" to the $\sigma_{\text {тот }}$ data. Rather than indulging in this inconsistent use of the deep inelastic structure functions, we have checked that our results on neutrino-mixing parameters are insensitive to this kind of variations of the input.

An important quantity in the zenith angle analysis is the average scattering angle between the lepton and the parent neutrino. In Fig. 10, we show the average angle for quasielastic events as a function of the lepton energy. This curve is in perfect agreement with that obtained by the SK Collaboration $[29,30]$.

\section{APPENDIX C: GEOMETRICAL ACCEPTANCES}

The SuperKamiokande detector is a cylinder of height $H=36.2 \mathrm{~m}$ and radius $R=16.9 \mathrm{~m}$, of fiducial volume $V$ $=\pi(R-2 \mathrm{~m})(H-4 \mathrm{~m})$. Let a point within the detector be labeled by $y$, the height from the bottom plane; $z$, the distance from the axis; and $x$, a third Cartesian coordinate. Let 


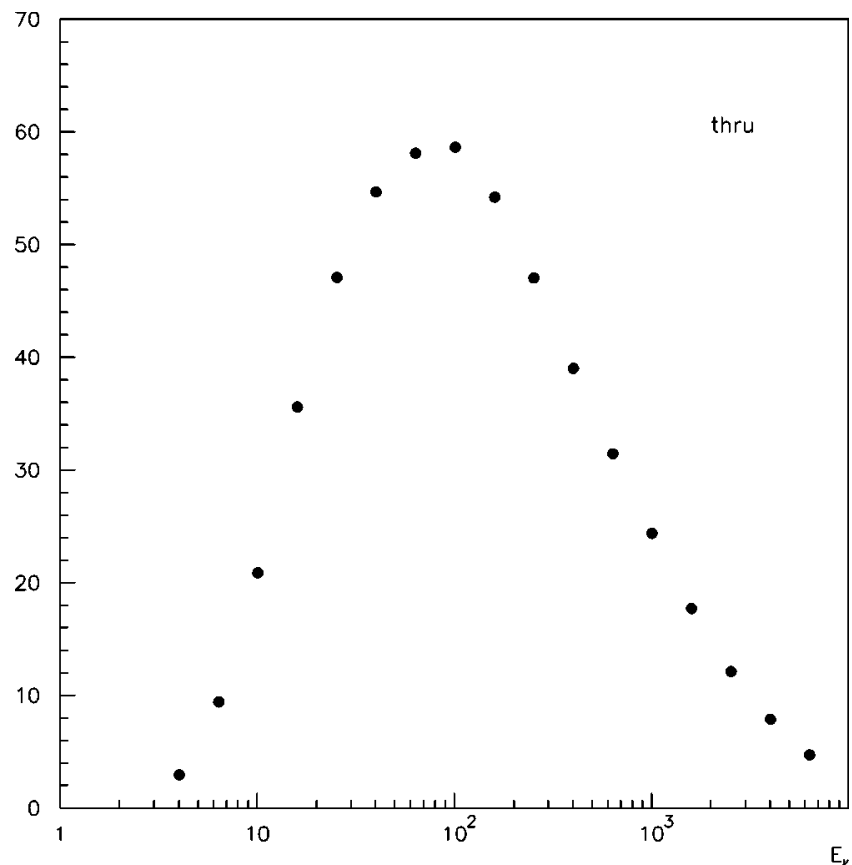

FIG. 12. Parent $\nu$ energies for through-going muons.

$d(x, y, z)$ be the minimum distance from a point in the detector to its wall, let $s_{\mu}, c_{\mu}, t_{\mu}$ be the sine, cosine, and tangent of the muon's azimuthal angle and let $E_{\text {min }}=0.7 \mathrm{GeV}$ be the energy of a muon giving a $2.6 \mathrm{~m}$ track in water.

We find

$$
\begin{aligned}
\mathrm{FC}\left(E_{\mu}, c_{\mu}\right)= & \frac{2}{V} \int_{0}^{R} d z \int_{0}^{2 \sqrt{R^{2}-z^{2}}} d x \int_{0}^{H} d y \Theta[d(x, y, z)-2 \mathrm{~m}] \\
& \times\left\{\Theta\left[2 \sqrt{R^{2}-z^{2}}-x-y\left|t_{\mu}\right|\right]\right. \\
& \times \Theta\left[y-R_{w}\left(E_{\mu}\right)\left|c_{\mu}\right|\right]+\Theta\left[2 \sqrt{R^{2}-z^{2}}-x\right. \\
& \left.\left.-R_{w}\left(E_{\mu}\right)\left|s_{\mu}\right|\right] \Theta\left[y\left|t_{\mu}\right|+x-2 \sqrt{R^{2}-z^{2}} \mid\right]\right\},
\end{aligned}
$$
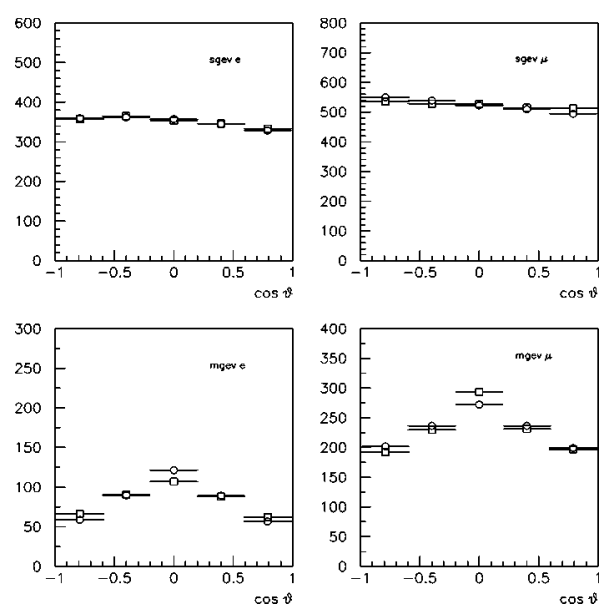

FIG. 13. Zenith-angle distributions of $e, \mu$ sgev, and mgev samples. The squares are the SK Monte Carlo results and the circles are our predictions, both for the no-oscillation hypothesis. The total areas under the curves are normalized to be the same.

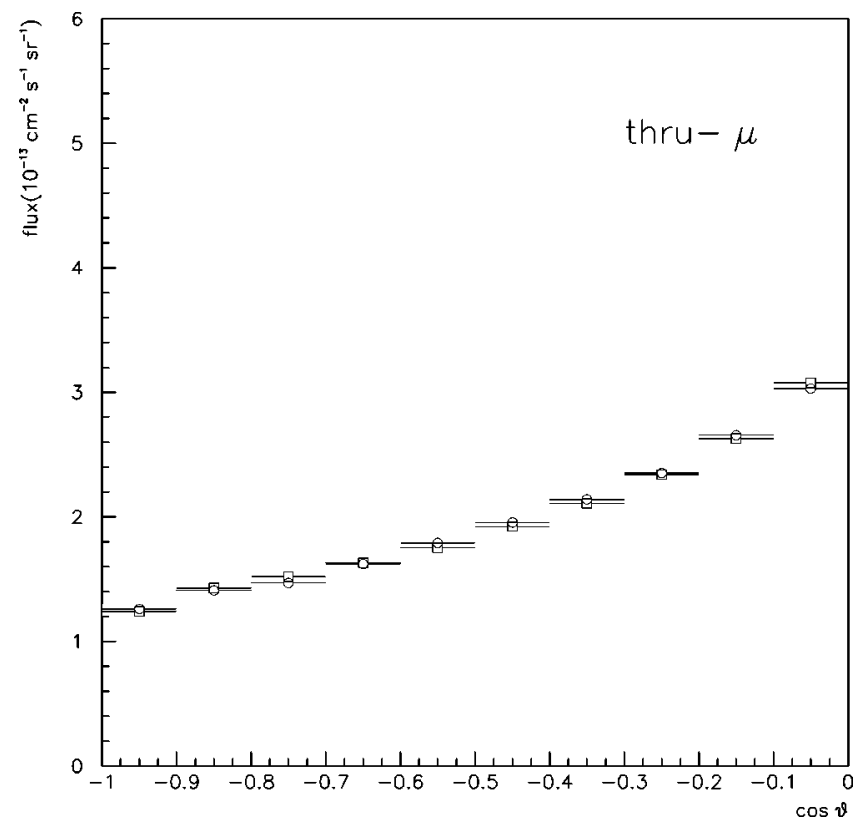

FIG. 14. Zenith-angle distributions of through-going muons for the nonoscillation hypothesis. The squares are SK Monte Carlo results and the circles are our predictions.

$$
\begin{aligned}
\operatorname{PC}\left(E_{\mu}, c_{\mu}\right)= & \frac{2}{V} \Theta\left[R_{w}\left(E_{\mu}\right)\right. \\
& \left.-R_{w}\left(E_{\text {min }}\right)\right] \int_{0}^{R} d z \int_{0}^{2 \sqrt{R^{2}-z^{2}}} d x \int_{0}^{H} d y \Theta \\
& \times[d(x, y, z)-2 \mathrm{~m}]\left\{\Theta \left[2 \sqrt{R^{2}-z^{2}}-x-y\right.\right. \\
& \left.\times\left|t_{\mu}\right|\right] \Theta\left[R_{w}\left(E_{\mu}\right)\left|c_{\mu}\right|-y\right]+\Theta\left[R_{w}\left(E_{\mu}\right)\left|s_{\mu}\right|\right. \\
& \left.\left.-2 \sqrt{R^{2}-z^{2}}+x\right] \Theta\left[y\left|t_{\mu}\right|+x-2 \sqrt{R^{2}-z^{2}} \mid\right]\right\},
\end{aligned}
$$

where the range of muons in water that we use, $R_{w}\left(E_{\mu}\right)$, can be obtained from the expressions in [4].

A muon produced with energy $E_{\mu}$, after traveling a distance $l$ in rock material, has an energy $E_{\mu}^{\prime}=R_{r}^{-1}\left[R_{r}\left(E_{\mu}\right)\right.$ $-l]$; its remaining range in water is $l_{w}\left(E_{\mu}, l\right)=R_{w}\left(E_{\mu}^{\prime}\right)$. The effective area for through-going muons of Eq. (20) is then given by

$$
\begin{aligned}
\mathrm{A}\left(E_{\mu}, c_{\mu}\right)= & \frac{2}{R_{r}\left(E_{\mu}\right)-R_{r}\left(E_{\text {min }}^{\prime}\right)} \int_{0}^{R_{r}\left(E_{\mu}\right)-R_{r}\left(E_{\text {min }}^{\prime}\right)} \\
& \times d l \int_{0}^{R} d z\left\{| s _ { \mu } | \int _ { 0 } ^ { H } d y \Theta \left[l_{w}\left(E_{\mu}, l\right)\right.\right. \\
& -\operatorname{Min}\left(2 \sqrt{\left.\left.\frac{R^{2}-z^{2}}{\left|s_{\mu}\right|}, \frac{[H-y]}{\left|c_{\mu}\right|}\right)\right]}\right. \\
& \times \Theta\left[\operatorname{Min}\left(2 \sqrt{\frac{R^{2}-z^{2}}{\left|s_{\mu}\right|}}, \frac{[H-y]}{\left|c_{\mu}\right|}\right)-7 \mathrm{~m}\right]
\end{aligned}
$$




$$
\begin{aligned}
& +\left|c_{\mu}\right| \int_{0}^{2 \sqrt{R^{2}-z^{2}}} d x \Theta\left[l_{w}\left(E_{\mu}, l\right)\right. \\
& -\operatorname{Min}\left(2 \sqrt{\left.\left.\frac{R^{2}-z^{2}-x}{\left|s_{\mu}\right|}, \frac{H}{\left|c_{\mu}\right|}\right)\right]}\right. \\
& \left.\times \Theta\left[\operatorname{Min}\left(2 \sqrt{\frac{R^{2}-z^{2}-x}{\left|s_{\mu}\right|}}, \frac{H}{\left|c_{\mu}\right|}\right)-7 \mathrm{~m}\right]\right\},
\end{aligned}
$$

where for the range of muons in rock $R_{r}\left(E_{\mu}\right)$, we have used the results in [17].

All of the above expressions can be integrated explicitly, but the analytical results are not brief, or useful.

\section{APPENDIX D: COMPARISON WITH THE SK MONTE CARLO SIMULATION}

We have not included in this analysis any nongeometrical detection efficiencies, as discussed in Sec. III. We have normalized the number of events in each data sample to the corresponding number in the SK Monte Carlo simulation for the nonoscillation hypothesis. This is tantamount to the use of an efficiency function which is not a function of energy within each sample. A nontrivial check that this is indeed a sensible approximation is to compare the parent neutrino energy distributions in the different data samples with those worked out by the SK team. These distributions are defined as the azimuthally averaged neutrino flux weighted with the integrated neutrino cross section and with the selection function for the various sgev and mgev data samples:

$$
\begin{aligned}
P_{s, l}\left(E_{\nu}\right) \propto & d c_{\nu} d E_{l} d c_{\beta} d \phi \mathrm{Th}_{\mathrm{s}, \mathrm{l}}\left(E_{l}, c_{l}\right) \\
& \times\left[\sigma\left(E_{\nu}, E_{l}, c_{\beta}\right) \frac{d \Phi_{\nu}}{d E_{\nu} d c_{\nu}}\right. \\
& \left.+\bar{\sigma}\left(E_{\nu}, E_{l}, c_{\beta}\right) \frac{d \Phi_{\nu}}{d E_{\nu} d c_{\nu}}\right] .
\end{aligned}
$$

All of the symbols in this expression have already been defined. Our results, shown in Figs. 11 and 12, are in good agreement with those obtained by SK [31].

In Figs. 13 and 14 we compare the zenith angle dependence obtained in our calculation for the nonoscillation hypothesis with the predictions of the SK Monte Carlo simulation [30]. The agreement is again rather good.
[1] Y. Fukuda et al., Phys. Lett. B 433, 9 (1998); Phys. Rev. Lett. 81, 1562 (1998); 82, 2644 (1999); Phys. Lett. B 467, 185 (1999); T. Kagita, in Proceedings of the XVIIIth International Conference on Neutrino Physics and Abstrophysics, Takayama, Japan, 1998.

[2] B. Pontecorvo, Zh. Éksp. Teor. Fiz. 53, 1717 (1967) [Sov. Phys. JETP 26, 984 (1968)].

[3] R. Davis et al., Phys. Rev. Lett. 20, 1205 (1968); B. T. Cleveland et al., Astrophys. J. 496, 505 (1998); T. Kirsten, in XVIII Conference on Neutrino Physics and Astrophysics, Takayama (Japan) 1998; SAGE Collaboration, Dzh. N. Abdurashistov et al., Nucl. Phys. B (Proc. Suppl.) 77, 20 (1999); Kamiokande Collaboration, Y. Fukuda et al., Phys. Rev. Lett. 77, 1683 (1996); Super-Kamiokande Collaboration, Y. Fukuda et al., ibid. 82, 1810 (1999); M. Smy, Super-Kamiokande Collaboration, hep-ex/9903034.

[4] Particle Data Group, C. Caso et al., Eur. Phys. J. C 3, 1 (1998).

[5] O. Yasuda, Phys. Rev. D 58, 091301 (1998); C. Giunti, C. W. Kim, and M. Monteno, Nucl. Phys. B521, 3 (1998); G. L. Fogli et al., Phys. Rev. D 59, 033001 (1999); hep-ph/9904465; R. Barbieri et al., J. High Energy Phys. 12, 017 (1998); V. Barger and K. Whisnant, Phys. Rev. D 59, 093007 (1999).

[6] Q. Y. Liu and A. Yu. Smirnov, Nucl. Phys. B524, 505 (1998); V. Barger, K. Whisnant, and T. Weiler, Phys. Lett. B 427, 97 (1998); S. C. Gibbons, R. N. Mohapatra, S. Nandi, and A. Raychaudhuri, ibid. 430, 296 (1998); M. C. Gonzalez-Garcia, et al., Nucl. Phys. B543, 3 (1999); D. Dooling, et al., Phys. Rev. D 61, 073011 (2000).

[7] G. L. Fogli et al., Phys. Rev. D 59, 033001 (1999); hep-ph/ 9904465.
[8] N. Hata and P. Langacker, Phys. Rev. D 56, 6107 (1997); J. N. Bahcall, P. I. Krastev, and A. Yu. Smirnov, ibid. 58, 096016 (1998); 60, 093001 (2000); R. Barbieri et al., J. High Energy Phys. 12, 017 (1998); P. C. de Holanda et al., Phys. Rev. D 60, 093010 (1999); M. C. Gonzalez-Garcia et al., Nucl. Phys. B573, 3 (2000); G. L. Fogli et al., Phys. Rev. D 62, 013002 (2000).

[9] L. Wolfenstein, Phys. Rev. D 17, 2369 (1978); 20, 2634 (1979); S. P. Mikheyev and A. Yu Smirnov, Yad. Fiz. 42, 1441 (1985) [Sov. J. Nucl. Phys. 42, 913 (1985)].

[10] O. L. G. Peres and A. Y. Smirnov, Phys. Lett. B 456, 204 (1999); A. Strumia, J. High Energy Phys. 04, 026 (1999).

[11] J. Pantaleone, Phys. Rev. D 49, 2152 (1994); Phys. Rev. Lett. 81, 5060 (1998); C. Giunti, C. W. Kim, and M. Monteno, Nucl. Phys. B521, 3 (1998); G. L. Fogli, et al., Phys. Lett. B 425, 341 (1998), and references therein; A. Y. Smirnov, hep-ph/9811296.

[12] PREM model by A. M. Diezwonski and D. L. Anderson, Phys. Earth Planet. Inter. 25, 297 (1981).

[13] R. N. Mohapatra and S. Nussinov, Phys. Lett. B 346, 75 (1995); P. F. Harrison et al., ibid. 349, 137 (1995); 396, 186 (1997); C. Giunti et al., ibid. 352, 357 (1995); P. F. Harrison, D. H. Perkins, and W. G. Scott, ibid. 458, 79 (1999); R. Foot, R. R. Volkas, and O. Yasuda, ibid. 433, 82 (1998); G. L. Fogli et al., hep-ph/9906450.

[14] M. Apollonio et al., Phys. Lett. B 420, 397 (1998); 466, 415 (1999).

[15] Y. Declais et al., Phys. Lett. B 338, 383 (1994).

[16] A. Donini et al., Nucl. Phys. B574, 23 (2000).

[17] M. Takahata, Ph.D. thesis, Niigata University, 1999.

[18] V. Agrawal, T. K. Gaisser, P. Lipari, and T. Stanev, Phys. Rev. 
D 53, 1314 (1996). We used the FORTRAN code provided by the authors.

[19] We warmly thank K. Scholberg for providing us with the official SK data, also shown in various talks: see, for instance, [30].

[20] A. A. Hahn et al., Phys. Lett. B 218, 365 (1989); K. Schreckenbach et al., Phys. Lett. 160B, 325 (1985); P. Vogel et al., Phys. Rev. C 24, 1543 (1981).

[21] P. Vogel and J. F. Beacom, Phys. Rev. D 60, 053003 (1999).

[22] A. De Rújula, M. B. Gavela, and P. Hernández, Nucl. Phys. B547, 21 (1999).

[23] C. H. Llewellyn Smith, Phys. Rep. 3C, 261 (1972).

[24] J. S. Bell and C. Llewellyn Smith, Nucl. Phys. B28, 317 (1971).
[25] M. Glück, E. Reya, and A. Vogt, Z. Phys. C 67, 433 (1995).

[26] O. Nachtmann, Nucl. Phys. B63, 237 (1973); A. De Rújula, H. Georgi, and H. D. Politzer, Phys. Rev. D 10, 2041 (1974).

[27] A. De Rújula, H. Georgi, and H. D. Politzer, Ann. Phys. (N.Y.) 103, 315 (1977).

[28] A. D. Martin et al., Eur. Phys. J. C 4, 364 (1998); 4, 463 (1998).

[29] K. Okumura, Ph.D. thesis, University of Tokyo, 1999.

[30] Y. Hayato, talk on Super-Kamiokande results at the Lyon workshop on the Neutrino Factory (NuFact99).

[31] K. Scholberg (private communication).

[32] Talk by T. Kajita for SuperKamiokande collaboration at PASCOS '99. For the last update of the analysis see H. Sobel, talk at Neutrino '00. 